\title{
Computer Geometry Applied to Locus Approach to Fire Stations
}

\author{
MASAHIRO MORITA \\ Department of Applied Mathematics, Faculty of Science \\ Science University of Tokyo \\ 1-3 Kagurazaka, Shinjuku-ku, Tokyo 162 Japan \\ YUKIO YAMAUCHI \\ Research and Development Laboratories \\ Hochiki Corporation \\ 246 Tsuruma, Machida-shi, Tokyo 194 Japan
}

\begin{abstract}
The diagram of influence for the fire-extinguishing activity of a fire station is represented by various Voronoi diagrams of the fire station. The ordinary Voronoi diagram, the network Voronoi diagram, high order network Voronoi diagram and the weighted network Voronoi diagram are introduced and defined for use in fire-fighting strategy. The Voronoi diagrams of the fire stations have been calculated and drawn on the personal computer from the actual city-map data of the metropolitan district in Japan. The reasonable scopes of influence for the fire-fighting of the fire stations are represented by the ordinary Voronoi diagram and the locus problems of the fire stations in the provincial towns may be estimated by the calculational result of ordinary Voronoi diagram. However, the locus problem in the metropolitan area must be estimated by the network Voronoi diagram in due consideration of the traffic congestion. In the fire-fighting strategy, the calculational results of the high order weighted network Voronoi diagram of the fire station may be capable to the dynamic allocations of the fire engines from the fire station to fire scene and to other fire station at the traffic snarl and the lane loading in the metropolitan district.
\end{abstract}

KEYWORDS : Voronoi diagram; Voronoi polygon; Ordinary Voronoi diagram; Network Voronoi diagram; High order network Voronoi diagram; Weighted network Voronoi diagram; fire station; fire-fighting strategy

\section{INTRODUCTION}

It is very important to discuss the activities of fire suppression at the fire occurred for the minimizing damage of human life and property. The fire is able to be extinguished by few of those present at the early stage fire occurred such as the fire before flashover. However, after flashover professional fire fighters are essential. Therefore the fire engines 
must arrive at the fire scene as quickly as possible.

In this paper, we introduce methods for estimation of the locus approach to the fire station in the district town applied to the Voronoi diagram $([1],[2])$ by computational geometry. This paper describes the ordinary Voronoi diagram method of fire station, which needs only the relative loci of fire stations and boundary locations of the calculational region in the map, and the network Voronoi diagram $([3],[4])$ of fire station applied to the traffic road in the metropolitan area. Moreover we have introduced the weighted network Voronoi diagram of fire station with due regard to lane loading, one-way streets, traffic snarls and speed limits for a dynamic schedule of allocation of fire engines. We have proposed the high order network Voronoi diagram of fire station from the point of view in the fire-fighting strategy. Namely the fire fighters in the fire engines belonging to the few fire stations which are the first, second and so on in order of proximate to the fire scene, turn out for and the fire engines belonging to these fire stations must be on constant alert for other fires in the precautious area of each fire station.

\section{FUNDAMENTAL CONSIDERATIONS AND CALCULATIONAL RE- SULTS}

The fire-fighting area of each fire station is important from the fire-fighting strategical point of view. We would itroduce the methods for preparation of the nearest fire-fighting area from each fire station using Voronoi Diagram algorithm.

\subsection{Application of Ordinary Voronoi Diagram of Fire Station}

We consider a finite number $n$ points, which are the numbers of fire station, in the Euclidean plane such as the city map, and assume that $2 \leq n<\infty$. The $n$ points are labelled by $p_{1}, \ldots, p_{n}$ with the Cartesian coordinates $\left(x_{1}, y_{1}\right), \ldots,\left(x_{n}, y_{n}\right)$ or location vectors $x_{1}, \ldots, x_{n}$ which are represented in the relative locus of the fire station in the map. The $n$ points are distinct in the sense that $\boldsymbol{x}_{\boldsymbol{i}} \neq \boldsymbol{x}_{j}$ for $i \neq j, i, j \in I_{n}=\{1, \ldots, n\}$. Let $p$ be an arbitrary point, such as the fire scene, in the Euclidean plane with coordinates $(x, y)$ or a location vector $\boldsymbol{x}$. Then the Euclidean distance from $p$ to $p_{\boldsymbol{i}}$ is given by $d\left(p, p_{i}\right)=\left\|\boldsymbol{x}-\boldsymbol{x}_{i}\right\|_{2}$. If $p_{i}$ is the nearest point from $p$ or $p_{i}$ is one of the nearest points from $p$, we have the relation $\left\|\boldsymbol{x}-\boldsymbol{x}_{i}\right\|_{2} \leq\left\|\boldsymbol{x}-\boldsymbol{x}_{j}\right\|_{2}$ for $j \neq i, j \in I_{n}$.

\subsubsection{Setting of the Application of Ordinary Voronoi Polygon to Locus Prob- lem of Fire Station}

Let a set of the fire station in the map $P=\left\{p_{1}, \ldots, p_{n}\right\} \subset \mathbf{R}^{2}$. We call the region given by

$V\left(p_{i}\right)=\left\{\boldsymbol{x} \mid\left\|\boldsymbol{x}-\boldsymbol{x}_{i}\right\|_{2} \leq\left\|\boldsymbol{x}-\boldsymbol{x}_{j}\right\|_{2}\right.$ for $\left.j \neq i, j \in I_{n}\right\}$

the ordinary Voronoi polygon of fire station associated with $p_{i}$. 
2.1.2 Setting of the Problem for Application of Ordinary Voronoi Diagram to Locus Problem of Fire Station

The set given by

$V_{O}(P)=\left\{V\left(p_{1}\right), \ldots, V\left(p_{\mathfrak{n}}\right)\right\}$

the ordinary Voronoi diagram of fire station generated by $P$.

The ordinary Voronoi polygon $V\left(p_{i}\right)$ is defined as the shortest fire fighting area $\left(s\left(p_{i}\right)\right)$ of the fire station $p_{i}$. The ordinary Voronoi diagram of fire station in the metropolitan area in Japan is shown the broken line in Figure 1.

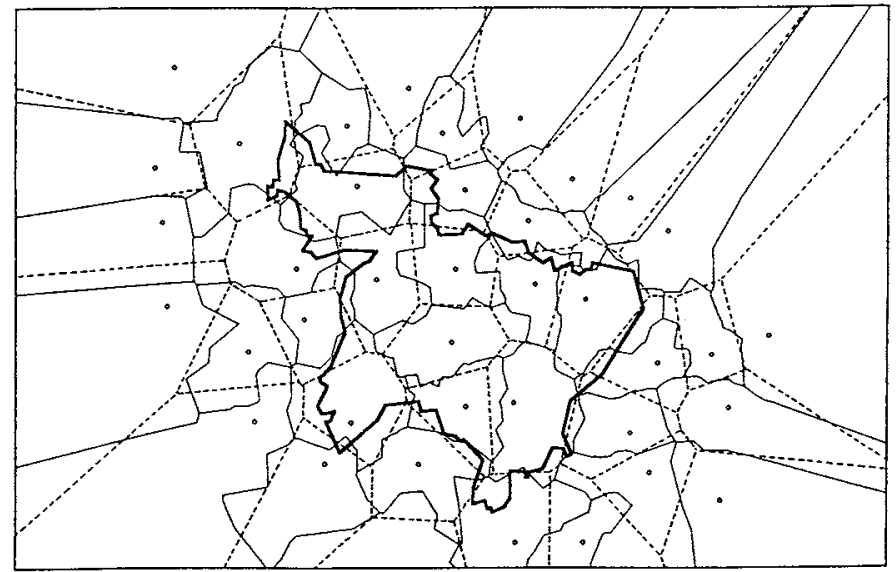

FIGURE 1 : Ordinary Voronoi diagram and network Voronoi diagram of fire station in the metropolitan area.

\subsection{Application of Network Voronoi Diagram of Fire Station}

The arbitrary locus point in the $s\left(p_{i}\right)$ is chosen the shortest distance in a straight line between this locus point and fire station among other fire stations. However, to deal with an ordinary Voronoi diagram of fire station in a actual metropolitan street system, it may be worth formulating a Voronoi diagram on a general network, because the fire fighters riding in the fire trucks motor to the fire scene on the street. Therefore we need introduce the new Ordinary Voronoi Diagram of Fire Station using the road system such as the Network Voronoi Diagram of Fire Station.

We consider a planar geometric graph $G(N, L)$ consisting of a set of the nodes $N=$ $\left\{q_{1}, \ldots, q_{m}\right\}$ which denote the intersections in the Euclidean plane map where $m$ is a 
number of intersections, and a set of links $L=\left\{l_{1}, \ldots, l_{k}\right\}$ which denote streets where $k$ is a number of streets in the map, which form a connected component. We also consider a set of distinct points such as fire stations $P=\left\{p_{1}, \ldots, p_{n}\right\}$ on a link in $L$ or a node in $N$. We assume that $G(N, L)$ is a non-directed graph (the extension to a directed graph is not difficult) made by the part of the metropolitan map in Japan as shown in Figure 2; where the closed thick line is the district border, the open circles are the fire stations, and the medium and thin lines are traffic roads. On $G(N, L)$ we define the distance, which is called the network distance and denote it by $d_{n}\left(p, p_{i}\right)$, from a point $p$ to $p_{i}$ on a link in $L$ or a node in $N$ by the length of the shortest path from $p$ to $p_{i}$. Let $r$ be also an arbitrary point in the Euclidean plane with coordinates $(x, y)$ or location vector $\boldsymbol{x}$. Then the Euclidean distance from a point $r$ to a point $p_{i}$ on the $G(N, L)$ is given by $d\left(r, p_{i}\right)=\left\|\boldsymbol{x}-\boldsymbol{x}_{\boldsymbol{i}}\right\|_{2}$. We call a geometric graph $G(N, L)$ with the network distance a network, and denote it by $N(N, L)$.

We consider a Euclidean plane map $S$ included the node set $N$, the link set $L$, and the fire station set $P$ in the map, called a plane of a network $N(N, L)$.

\subsubsection{Setting of the Application of Access Distance}

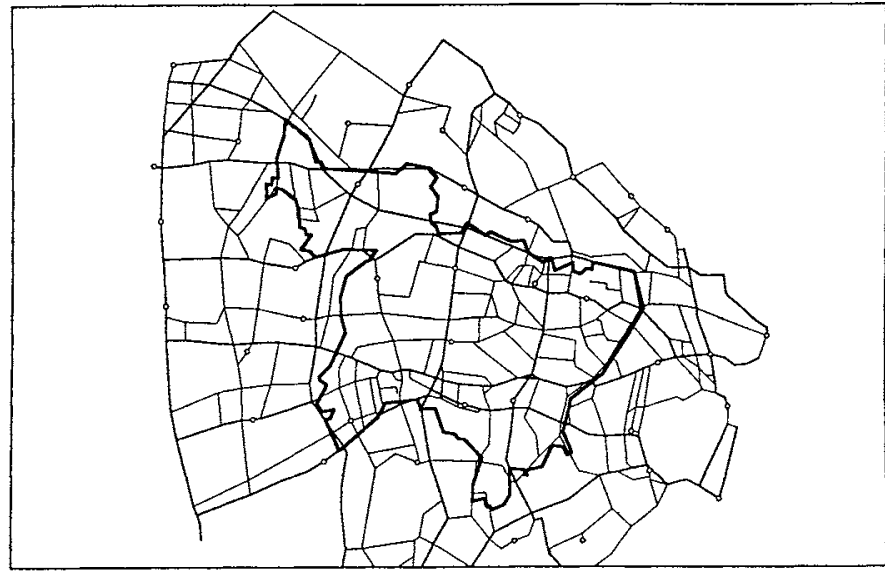

FIGURE 2 : The planar geometric graph $G(N, L)$ for streets and fire stations map of the metropolitan area.

We first assign a point $r$ in $S$ to the nearest point, called the access point, on a link of $L$. Second the access point is assigned to the shortest point $p_{i}$ in $\mathrm{P}$ with respect to the network distance. To be precise, let $a(p)$ be the nearest point on $L$ from $r$.

$d_{a}(r, a(p))=\left\|\boldsymbol{x}-\boldsymbol{x}_{a}\right\|_{2}=\min _{\boldsymbol{u}}\left\{\|\boldsymbol{x}-\boldsymbol{u}\|_{2} \mid \boldsymbol{u} \in L\right\}$

Where $\boldsymbol{x}, \boldsymbol{x}_{\boldsymbol{a}}$ and $\boldsymbol{u}$ are the location vectors of points $r, a(p)$ and arbitrary point on $L$, respectively. We call the point $a(p)$ the access point of $p$ to $N(N, L)$, and the distance 
$d_{a}(r, a(p))$ the access distance.

\subsubsection{Setting of the Network Access Distance}

In terms of the access distance and the network distance, the distance which is called the network access distance from a point $r$ in Euclidean plane map to a point $p_{i}$ in $L$ or $N$ is written as follows.

$d_{n a}\left(r, p_{i}\right)=d_{a}(r, a(p))+d_{n}\left(a(p), p_{i}\right)$

\subsubsection{Setting of the Application of Network Voronoi Polygon to Locus problem} of Fire Station

With the distance of equation, we define the network Voronoi polygon of fire station as follows.

$V_{n}\left(p_{i}\right)=\left\{r \mid d_{n a}\left(r, p_{i}\right) \leq d_{n a}\left(r, p_{j}\right), j \neq i, i, j \in I_{n}\right\}$

2.2.4 Setting of the Application of Network Voronoi Diagram to Locus Problem of Fire Station

The network Voronoi diagram of a fire station is generated by $P$ as following equation.

$V_{N}(P)=\left\{V_{n}\left(p_{1}\right), \ldots, V_{n}\left(p_{n}\right)\right\}$

The network Voronoi polygon of a fire station means $V_{n}\left(p_{i}\right)$ is defined as the fire fighting area with the shortest distance $\left(s\left(p_{i}\right)\right)$ of a fire station $p_{i}$ among the other fire stations $p_{j}, j \neq i$. The network Voronoi diagram of fire station in the metropolitan area in Japan is shown the straight line in Figure 1.

\subsection{Application of High Oder Network Voronoi Diagram of Fire Station}

From the point of view of fire-fighting strategy, when a fire occurs in more than two places at the same time in the shortest fire fighting area of the fire station, the fire fighters not only must hasten to the fire scenes from this fire station, but also from other fire stations. Therefore in this case we have to introduce the high order network Voronoi diagram of fire station.

2.3.1 Setting of the Application of Order- $h$ Network Voronoi Polygon to Locus Problem of Fire Station

Let $A^{(h)}(P)$ be the set of all possible subsets consisting of $h$ elements out of $P$, i.e. $A^{(h)}(P)=\left\{P_{1}^{(h)}, \ldots, P_{i}^{(h)}, \ldots, P_{t}^{(h)}\right\}$, where $P_{i}^{(h)}=\left\{p_{i_{1}}, \ldots, p_{i_{h}}\right\}, p_{i j} \in P$, and $t={ }_{n} C_{h}$. In these terms, we define following form.

$V_{n}\left(P_{i}^{(h)}\right)=\left\{p \mid \max _{p_{h}}\left\{d_{a n}\left(p, p_{h}\right) \mid p_{h} \in P_{i}^{(h)}\right\} \leq \min _{p_{j}}\left\{d_{a n}\left(p, p_{j}\right) \mid p_{j} \in P \backslash P_{i}^{(h)}\right\}\right\}$

We call the set $V_{n}\left(P_{i}^{(h)}\right)$ the order-h network Voronoi polygon associated with $P_{i}^{(h)}$.

2.3.2 Setting of the Application of Order- $h$ Network Voronoi Diagram to Locus Problem of Fire Station

And the set of order $-h$ network Voronoi polygons, $V_{N}^{h}(P)$ is defined as follows. 
$V_{N}^{h}(P)=\left\{V_{n}\left(P_{1}^{(h)}\right), \ldots, V_{n}\left(P_{t}^{(h)}\right)\right\}$

We call the order- $h$ network Voronoi diagram generated by $P$.

The caluculational results of the first-order and third-order network Voronoi diagram of the fire station in the metropolitan area are shown in Figure 3.

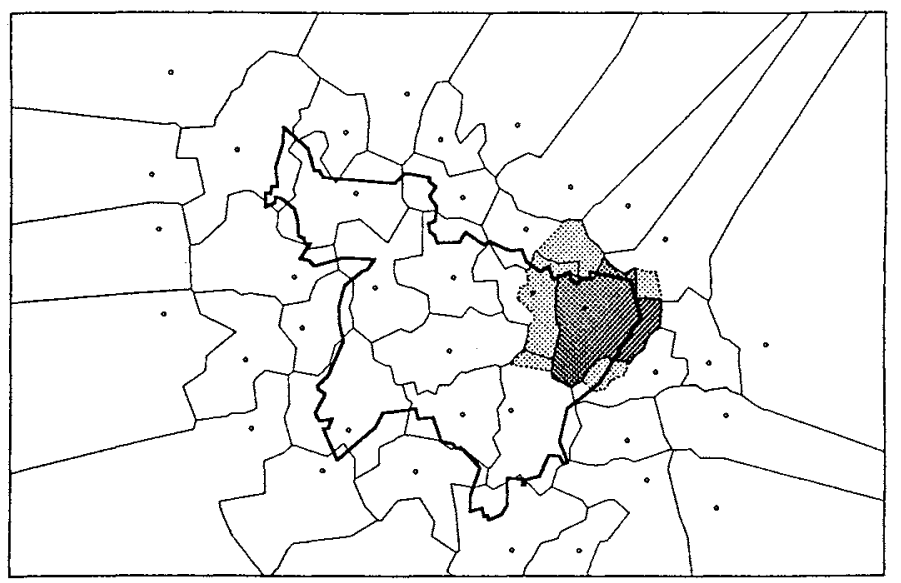

FIGURE 3 : First-order and third-order network Voronoi diagram of fire station in the metropolitan area.

\subsection{Application of Weighted Network Voronoi Diagram of a Fire Station}

As the fire engine passes along various streets, the fire engines speed will vary depending upon the width and the speed limit of the streets, and also upon traffic congestion. Therefore the time required for the fire engine to reach the fire scene instead of the dominance length must be adopted in the network Voronoi diagram.

In the network Voronoi diagram we implicitly assume that the network access distances, which are composed of the network distances and the access distances, are indifferent and have the same weight. Therefore we assume that the network distances between them have different weights reflecting the variable property and information of the streets We show the weighted network Voronoi diagram that takes the different weight in to account in terms of the weighted network distance.

We represent the weight by the set of the parameters, $W=\left\{w_{1}, w_{1_{b}} \ldots, w_{k_{f}}, w_{b}\right\}$, which are defined by the street informations and properties where $k$ denotes the number of the weight parameters corresponding to the number of streets and the number of weight 
parameters is $2 k$ for two-way streets and the traffic snarl on the street.

\subsubsection{Setting of the Weight parameter}

We consider the street $l_{s}$ in $L$ which connects the intersection $p_{s}^{a}$ in $N$ to the adjacent intersection $p_{s}^{b}$. We also consider a set of averaged speed $V_{v e l}=\left\{v_{1}, v_{l_{b}}, \ldots, v_{k_{f}}, v_{k_{b}}\right\}$ of the fire engine motoring between the $p_{s}^{a}$ to $p_{s}^{b}$ on the street $l_{s}$. We assume that the fire engine motors.

$$
\begin{cases}w_{s_{j}}\left(p_{s}^{a}, p_{s}^{b}\right)=v_{s}\left(p_{s}^{a}, p_{s}^{b}\right) / \max _{i_{j}}\left|v_{i_{j}}\left(p_{i}^{a}, p_{i}^{b}\right)\right|: \text { (going way) } \\ w_{s_{b}}\left(p_{s}^{a}, p_{s}^{b}\right)=v_{s_{b}}\left(p_{s}^{a}, p_{s}^{b}\right) / \max _{i_{j}}\left|v_{i_{j}}\left(p_{i}^{a}, p_{i}^{b}\right)\right|: \text { (returning way) }\end{cases}
$$

\subsubsection{Setting of the Weighted distance}

With this weight we define a weight distance $d_{w}\left(p_{s}^{a}, p_{s}^{b}\right)$ from the point $p_{s}^{a}$ to $p_{s}^{b}$ which is specified in the following form.

$\left\{\begin{array}{l}d_{w}\left(p_{s}^{a}, p_{s}^{b}\right)=w_{s_{j}}\left(p_{s}^{a}, p_{s}^{b}\right) \cdot d_{n}\left(p_{s}^{a}, p_{s}^{b}\right) \\ d_{w}\left(p_{s}^{b}, p_{s}^{a}\right)=w_{s_{b}}\left(p_{s}^{a}, p_{s}^{b}\right) \cdot d_{n}\left(p_{s}^{a}, p_{s}^{b}\right)\end{array}\right.$

We define again the weight distance $d_{w}\left(p_{s}, p_{s}^{b}\right)$ from the point $p_{s}$ on the street $l_{s}$ in the street set $L$ to the nearest intersection $p_{s}^{b}$ on the same street $l_{s}$ which specified with the weight parameters $w_{s_{f}}$ and $w_{s_{b}}$ of the two-way street $l_{s}$ as follows.

$\begin{cases}d_{w}\left(p_{s}, p_{s}^{b}\right)=w_{s_{f}}\left(p_{s}, p_{s}^{b}\right) \cdot d_{n}\left(p_{s}, p_{s}^{b}\right) & : \text { (going way) } \\ d_{w}\left(p_{s}, p_{s}^{b}\right)=w_{s_{b}}\left(p_{s}, p_{s}^{b}\right) \cdot d_{n}\left(p_{s}, p_{s}^{b}\right) & : \text { (returning way) }\end{cases}$

Where the motoring direction of the fire engine is represented by the nearest intersection of the point $p_{s}$.

\subsubsection{Setting of the Weighted Network Distance}

We furthermore define the weighted network distance $d_{w n}\left(p_{s}, p_{f}\right)$ from the point $p_{s}$ on the street $l_{s}$ to the fire station $p_{f}$ in the street set $L$ or the intersection set $N$ by the weighted length of the shortest path from $p_{s}$ to $p_{f}$ which described as follows.

$d_{w n}\left(p_{s}, p_{f}\right)=d_{w}\left(p_{s}, p_{s}^{b}\right)+\sum_{i=2}^{c} d_{w}\left(p_{s_{i}}^{a}, p_{s_{i}}^{b}\right)+d_{w}\left(p_{s_{f}}^{a}, p_{f}\right)$

Where the rote from the point $p_{s}$ to $p_{f}$ is in order of $l_{s}, l_{2}, \ldots, l_{c}$, and $l_{f}$. The point $p_{s}^{b}$ is just the same point $p_{s_{2}}^{a}, p_{s_{i-1}}^{b}=p_{s_{i}}^{a}(i=3, \ldots c)$ and $p_{s_{c}}^{b}=p_{f}$.

We consider again the Euclidean plane metropolitan map $S$ included the intersection set $N$, the street set $L$ and fire station set $P$, called a metropolitan network, and we assume the direct graph because of including the one way street in $L$.

\subsubsection{Setting of the Weighted Network Access Distance}

The weighted network access distance from the arbitrary point $r$ to the fire station $p_{f}$ in $S$ is written by the weighted network distance and the access distance as follows.

$d_{w n a}\left(r, p_{f}\right)=d_{a}\left(r, a\left(p_{s}\right)\right)+d_{w n}\left(a\left(p_{s}\right), p_{f}\right)$ 
2.4.5 Setting of the Application of Weighted Network Voronoi Polygon to Locus Problem of Fire Station)

With the weighted network access distance of equation, we define the weighted network Voronoi diagram of fire station as follows.

$V_{w n}\left(p_{i}\right)=\left\{r \mid d_{w n a}\left(r, p_{i}\right) \leq d_{w n a}\left(r, p_{j}\right), j \neq i, i, j \in I_{n}\right\}$

2.4.6 Setting of the Application of Weighted Network Voronoi Diagram to Locus Problem of Fire Station

The weighted network Voronoi diagram of fire station is generated by $P$ as following equation.

$V_{W N}(P)=\left\{V_{w n}\left(p_{1}\right), \ldots, V_{w n}\left(p_{n}\right)\right\}$

The weighted network Voronoi polygon of fire station means the $V_{w n}\left(p_{i}\right)$ is defined as the fire fighting area with the shortest travel time $\left(s_{w}\left(p_{i}\right)\right)$ from fire station $p_{i}$ among the other fire stations $p_{j}, j \neq i$. The weighted network Voronoi diagram of fire stations in the metropolitan area in Japan is shown in Figure 4, where the average speed of the fire engine is assumed to $40 \mathrm{Km} / \mathrm{h}, 30 \mathrm{Km} / \mathrm{h}$ and $20 \mathrm{Km} / \mathrm{h}$ for first-class national roads, the main principal roads and other roads, respectively.

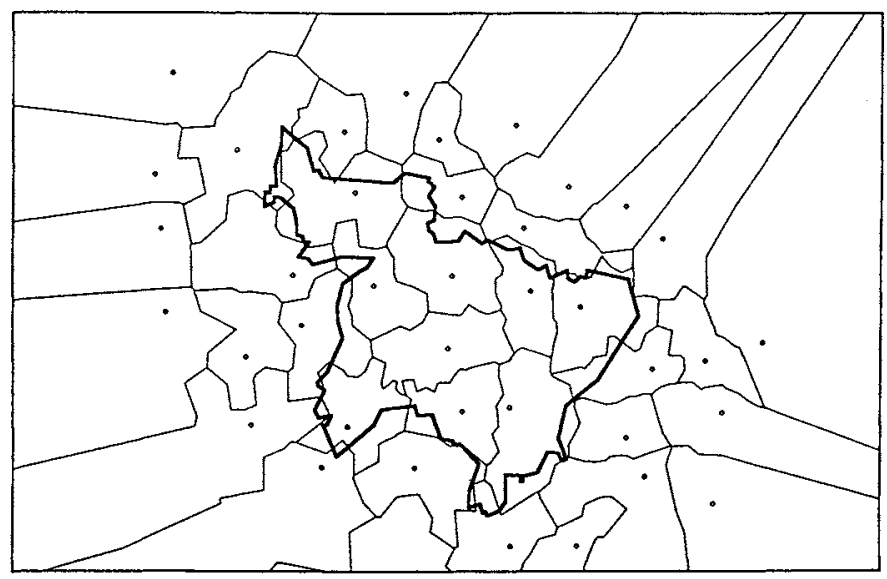

FIGURE 4 : Weighted network Voronoi diagram of fire station in the metropolitan area.

\section{DISCUSSIONS}

The ordinary Voronoi polygons of the fire station, the broken line, are much of the size as the network Voronoi polygons, the straight line, in Figure 1. The method of 
the ordinary Voronoi diagram has an advantage over the method of the network Voronoi diagram because of only the relational locus points of the fire stations in the map have to be input. However the network Voronoi diagram of the fire station must be considered for firefighting strategy. The methods of the network Voronoi diagram require immense amounts of input-data about the streets and intersections shown in Figure 2, and moreoverhave an enormous additional expense for updating data of them for the restructual planning. The method of the ordinary Voronoi diagram of the fire station is useful in city planning and for the fire fighting strategy of provincial towns.

The small difference between the fire fighting areas of turning out to fight which are the first and third order network Voronoi polygon of the fire station in the metropolitan area as shown in Figure 3 represent a well-planned fire-fighting strategy. The smaller fire-fighting area given by the Voronoi polygon, the fire-fighting area becomes smaller the conflagration hazard of the building. In fact, it is necessary to evaluate the time required by the fire engines rather than the distances from the fire stations to the fire scenes. Therefore we must discuss that how many hours the fire engine requires to the locus point of most requiring a lot of time from the fire station in the Voronoi polygon. And the fire-fighting superficial content, which corresponds to the (weighted) network Voronoi polygon, constructed by the administrative determined requiring time of the fire engine from the fire station to the fire scene needs to perfectly include in the Voronoi polygon. The method of the network Voronoi diagram of the fire station can be put to practical use for the activities of fire suppression by the fire department. Moreover the (high order) weighted network Voronoi diagram of the fire station is capable of dynamically allocating fire engines to fire scenes in the metropolitan area taking into account traffic snarls and the lane loading, but it is important to investigate traffic volume.

\section{REFERENCES}

1 G. Voronoi; Nouvelles applications des paramètres continus à la theórie des formes quadratiques, deuxième memoire, recherches sur les parallelloèdres primitifs; Journal für die Reine und Angewandte Mathematik, 134 (1908)

2 C.A. Rogers; Packing and Covering; Cambridge Tracts in Mathematics and Mathematical Physics 54 (1964)

3 M. Iri and T. Koshizuka; Computational Geometry and Geographical Information Processing; Kyoritu (1986) in Japanese

4 A. Okabe and H. Yomono; Statistical methods for evaluating the geometrical hierarchy of a network; Geographical Analysis 20-2 (1988) 
